\title{
Validación de constructo de componentes de agencia académica evaluados en web
}

Construct validation of academic agency components evaluated on a web platform

\author{
Sandra Castañeda F. ${ }^{1}$, Iván Pérez C., Rodrigo Peña D. \\ LABORATORIO DE EVALUACIÓN Y FOMENTO DEL DESARROLLO COGNITIVO Y EL APRENDIZAJE COMPLEJO \\ Universidad Nacional Autónoma de MÉxico \\ RECIBIDO 01/09/2016, AcEPTADO 02/11/2016
}

\begin{abstract}
RESUMEN
Con datos de 1361 estudiantes, validamos el metaevaluador web donde: a) el banco de estrategias de estudio y autorregulación usó 50 ítems calibrados para realizar análisis factoriales confirmatorios. Los resultados de sus bancos (estrategias cognitivas; de autorregulación de persona y de tarea y materiales) mostraron índices mayores de $.90 \mathrm{y}$ RMSEA menores de .07, con alfas de Cronbach $>0.83$. El ajuste entre los modelos teóricos y empíricos validaron sus constructos; b) el banco, epistemología personal, utilizó 12 ítems calibrados y su índice de ajuste confirma validez de constructo $(.90$, RMSEA $=.07)$ y c) el de comprensión de textos utilizó 18 reactivos calibrados, con validez convergente (CFI =.96; RMSEA $=.03$ ). Todos con una homogeneidad aceptable.
\end{abstract}

Palabras clave: Validación de constructo, agencia académica, aprendizaje académico autorregulado, evaluación en línea.

\begin{abstract}
Using data from 1361 students we validate the Meta Evaluador Web where: a) the bank of Study and Self-Regulation Strategies used 50 items calibrated for confirmatory factor analysis. The results of their banks (Cognitive Strategies; Person and Self-Regulation Task and Materials) showed higher rates to 0.90 and RMSEA less than 0.07, with Cronbach alpha's $>0.83$. The fit between the theoretical and empirical models validated their constructs; $b$ ) the Personal Epistemology bank, used 12 item calibrated and its adjustment index confirms construct validity $(0.90$, RMSEA $=0.07)$ and $\mathrm{c})$ Reading Comprehension bank used 18 calibrated items with convergent validity $(\mathrm{CFI}=0.96$; $\mathrm{RMSEA}=0.03)$, all of them, with an acceptable homogeneity.
\end{abstract}

Keywords: Construct validation, academic agency, self-regulated academic learning, on line measurement.

1 Posgrado, Facultad de Psicología, UNAM, México. E-mail: sandra@unam.mx 


\section{INTRODUCCIÓN}

En tanto las sociedades del conocimiento exigen a las instituciones de educación superior (IES) nuevos capitales culturales en sus egresados, más acordes con una sociedad en continua transformación, los educadores estamos llamados a revisar creencias, fundamentos teóricos y prácticas formativas en los que hemos basado la docencia. De la misma manera, a identificar, entender y fomentar los mecanismos que determinan, de múltiples maneras, los nuevos capitales culturales requeridos en una época en la que la emergencia de la revolución de las tecnologías de la información y la comunicación promueven, de suyo, la globalización en muchas facetas de las prácticas sociales, políticas, científicas y, desde luego, las educativas.

Los docentes hemos visto cómo el perfil de nuestros estudiantes ha cambiado en sus estilos de procesamiento -ahora guiados más por imágenes que por palabras-, con demandas de procesamiento sintético más que analítico; con sobrecargas cognitivas de naturaleza diversa sobre la memoria de trabajo y otros componentes atencionales e intencionales, así como también en los modos epistémicos, otrora impensables, en los que los estudiantes se ven requeridos a construir el conocimiento. Y es en este plano donde la cultura virtual y las redes sociales facilitan el aprendizaje colaborativo y ponen en entredicho la asimétrica relación estudiante-profesor, característica del paradigma pedagógico tradicional. Toca optimizar la idoneidad de la función de los docentes en educación superior, particularmente, para hacerlos estrategas en el desarrollo de los mecanismos del estudiante que fomentan una formación profesional de calidad.

Esta premisa requiere de una planeación coherente de los programas universitarios y un cambio de estructura donde el objetivo principal sea dotar a la sociedad civil de ciudadanos más autónomos y agentivos, capaces de mejorar el servicio o productos, pero, también, capaces de adaptarse flexible y creativamente a situaciones imprevisibles. En otras palabras, ciudadanos con capacidad de comprender el cuerpo de conocimientos, habilidades y valores en los que basa su actividad; que dominen las habilidades generales y específicas, suaves y técnicas, necesarias para desarrollar su actividad profesional y su propio crecimiento personal. Con estrategias para analizar problemas, construir o seleccionar las mejores soluciones posibles y, de manera importante, ser su propio agente en la transformación de lo establecido y de sí mismo, si es necesario. En resumen, ciudadanos con habilidades de razonamiento, de solución de problemas y con control emocional y volitivo para mejorar, profundizar y extender el alcance de sus capacidades.

Y dado que, hoy día, uno de los retos más importantes del sistema educativo es fomentar la autonomía en los estudiantes, resulta crucial fomentar su sentido de agencia. En el contexto formativo, este sentido es visto como un estado experiencial mediante el cual el estudiante hace uso de sus recursos (cognitivos, autorregulatorios, volitivos, epistemológicos, sociales y de regulación emocional, entre otros) para lograr metas individuales y sociales. La intencionalidad es su dispositivo central. 
En nuestra aproximación, el sentido de agencia académica es entendido como un fenómeno complejo en el que confluyen múltiples componentes que se articulan a todo lo largo de la trayectoria de los episodios de aprendizaje académico: desde la intención de lograr un resultado determinado hasta el resultado en sí mismo, dado que este genera la experiencia de agencia y la emoción concomitante (disfrute o confusión).

Durante las actividades que se realizan en los episodios de aprendizaje, los estudiantes son capaces de darse cuenta de que se "mueven" (o no) hacia un mejor aprendizaje; por ejemplo, pueden reconocer que haber logrado un premio o distinción por un desempeño académico sobresaliente se debe a que ellos se han movido hacia esa meta (son los dueños de la acción) y que, de alguna manera específica, se involucraron intencionalmente en una serie de acciones que causalmente se asociaron con la producción de esos resultados. Es decir, son los autores de tales acciones y, por lo tanto, los responsables de las resultantes (la experiencia de agencia).

Sin embargo, puede darse el caso de que aunque el estudiante se oriente hacia el reconocimiento académico, no se asuma como el dueño de la acción. Es decir, no se reconozca (ni se comprometa) como el que inicia, identifica, diversifica y mantiene acciones que lo llevan a conseguir lo que intenta lograr; en otras palabras, que no se reconozca como el autor que tradujo la intención en acción.

Y es con base en lo anterior que, en 2007, Castañeda propusó un modelo teórico de agencia académica que integró avances sobre mecanismos del aprendizaje académico autorregulado producidos en los últimos 30 años en diversos laboratorios, incluido el de ella. Estos avances constituyen, hoy día, un sólido marco de referencia para un mejor entendimiento de los mecanismos determinantes del aprendizaje académico autorregulado y pueden ser conceptuados como componentes de agencia académica.

$\mathrm{Y}$ dado que analizar la interacción inteligente requiere modelos sobre la complejidad de las situaciones reales, en nuestro caso, estas han sido representadas por componentes, de naturaleza variable, que interactúan entre sí y sirven como "recursos agentivos" al servicio de la intencionalidad del estudiante.

A partir de evidencias e instrumentación generadas en la línea de investigación de Castañeda sobre aprendizaje académico autorregulado (1980 a la fecha), se generó un macroproyecto multietápico cuyo interés fue identificar los efectos que todos los componentes juntos (y en interacción) tendrían sobre el aprendizaje y procesos asociados (Castañeda, 2007).

El Consejo Nacional de Ciencia y Tecnología (Conacyt) financió la primera etapa del macroproyecto (2007-2011) y, en 2014, Castañeda, Peñalosa y Austria reportaron una investigación en la que se pusieron a prueba algunos componentes de agencia académica, en tres contenidos de aprendizaje (motivación, aprendizaje y percepción) y dos modalidades de entrega (presencial y virtual). 
Las evidencias dejaron en claro las complejas estructuras generadas por las interrelaciones entre variables del estudiante, del contexto y del contenido que se configuran durante episodios de aprendizaje construidos. De esta manera, se mostraron efectos diferenciales derivados del nivel (alto o bajo) con el que los estudiantes movilizaron sus recursos cognitivos, autorregulatorios y de epistemología personal para aprender. Se asumió y constató que la riqueza de la base de conocimientos, los sistemas de creencias epistemológicas y las habilidades de autorregulación interactúan para que los individuos puedan desarrollar la capacidad de ejercer influencia personal sobre su ambiente y para jugar un papel activo en su autogestión (Castañeda, 2012).

En breve, en esta investigación se identificaron y validaron componentes que la literatura resaltaba como determinantes del aprendizaje académico. Así, y a partir de datos de 600 estudiantes de Psicología de siete instituciones formadoras mexicanas, se generó evidencia sobre heurísticas cognitivas, autorregulatorias y creencias epistemológicas como componentes de agencia. Estas dejaron en claro que: a) se generan complejas estructuras de interrelaciones entre variables del estudiante, del contenido y del contexto en función de la diversidad entre ellos; b) que hay efectos diferenciales estables, derivados del nivel de dominio (alto o bajo), con el que los estudiantes utilizaron, intencionalmente, sus recursos cognitivos, autorregulatorios y de epistemología personal para resolver lo solicitado; así, la riqueza de la base de conocimientos, el desarrollo epistemológico y el de los procesos autorregulatorios interactúan y afectan la experiencia de agencia con la que pueden influir personalmente y actuar un rol activo sobre su autogestión. En síntesis, se concreta la experiencia de agencia con la que el estudiante experimenta su papel autogestor.

Otro avance importante dentro de la investigación de agencia implica la buena calidad psicométrica y validez de constructo en los instrumentos generados/ adaptados específicamente para tal investigación. Conacyt premió la investigación como Caso de éxito en el área de Investigación Básica en 2012.

Dadas las necesidades de universidades masificadas y contando con la ventaja de tener a disposición instrumentos psicometrados, se identificó la oportunidad de crear derivación tecnológica de la instrumentación generada hasta ese momento. Y es en este punto en el que se ancla el objetivo del presente trabajo.

En este contexto, el objetivo fue validar los constructos que subyacen a los bancos de reactivos y establecer las estructuras factoriales entre las relaciones para cada uno de los bancos que integran este artefacto estandarizado de evaluación, al que hemos llamado Metaevaluador web de componentes de agencia académica.

Si bien, con anterioridad, los instrumentos fueron probados psicométricamente, este análisis se realizó cuando la entrega fue presencial. Sin embargo, las evidencias sugieren que hay diferencias de un contexto de entrega presencial a uno virtual, en vista de que este último plantea mayores demandas cognitivas y autorregulatorias al estudiante (Castañeda, Peñalosa y Austria, 2014). 
Asimismo, se asume que en este formato de entrega se potencializaría su impacto al liberar su aplicación de tiempos personales (disponibilidad de horario) y de ubicación geográfica (disponibilidad de lugar). Además de dar oportunidad al estudiante de experimentar acciones intencionales que evidencien cómo es que usan sus recursos para el desarrollo de cursos de acción en función de sus metas (Castañeda, 2012; Castañeda, Peñalosa y Austria 2014; Castañeda, Pérez y Peñalosa, 2014).

\section{MÉTODO}

\section{Participantes}

De una muestra mayor de estudiantes (1703), se seleccionaron 1361 casos, de diversas disciplinas y semestres, de ocho instituciones públicas y privadas de educación superior en México. Los criterios de inclusión fueron: a) estar inscrito en el semestre en curso en una universidad mexicana, b) aceptar participar voluntariamente y c) haber respondido íntegramente lo solicitado.

\section{Materiales}

El Metaevaluador web de componentes de agencia académica consiste en un portal en línea en el que se implementaron los instrumentos: Inventario de Estrategias de Estudio y Autorregulación (IEEA); Inventario de Epistemología Personal (IEP) y Prueba de Comprensión de Textos (CT).

\section{DESCRIPCIÓN DE LOS COMPONENTES QUE SE MIDEN EN EL METAEVALUADOR}

El primer componente que presenta el metaevaluador es el Inventario de Estrategias de Estudio y Autorregulación (IEEA). Su estructura está constituida por 52 reactivos tipo Likert, organizados en cuatro subescalas: a) estilos de adquisición de información (selectiva y generativa); b) estilos de recuperación de la información aprendida (ante tareas y ante exámenes); c) estilos de procesamiento de la información (convergente y divergente) y d) estilos de autorregulación (metacognitiva y metamotivacional), constituidos por tres componentes: los del estudiante (autoeficacia, contingencia percibida, autonomía percibida y orientación a la aprobación externa); los de la tarea de aprendizaje (orientación a dominio o a la ejecución); y, finalmente, los de los materiales, en cuanto a su evaluación y regulación. Una síntesis de la estructura del IEEA aparece en la figura 1. 
Figura 1. Inventario de Estrategias de Estudio y Autorregulación

\begin{tabular}{|c|c|c|c|c|}
\hline Área & Dimensión & Escala & Medida & Descripción \\
\hline \multirow{6}{*}{ 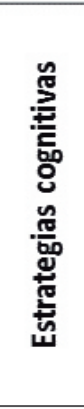 } & \multirow{2}{*}{$\begin{array}{c}\text { Estilos de } \\
\text { adquisición de } \\
\text { información }\end{array}$} & 1 & Selectivas & Estrategias de procesamiento superficial \\
\hline & & 2 & Generativas & Estrategias de procesamiento profundo \\
\hline & \multirow{2}{*}{$\begin{array}{c}\text { Estilos de } \\
\text { recuperación } \\
\text { de la } \\
\text { información } \\
\end{array}$} & 3 & Ante tareas & $\begin{array}{l}\text { Estilo para recuperar información en tareas } \\
\text { diversas }\end{array}$ \\
\hline & & 4 & Durante exámenes & $\begin{array}{l}\text { Estilo para recuperar conocimiento durante } \\
\text { evaluaciones diversas }\end{array}$ \\
\hline & \multirow{2}{*}{$\begin{array}{c}\text { Estilos de } \\
\text { procesamiento }\end{array}$} & 5 & Convergente & Reproducir la información aprendida \\
\hline & & 6 & Divergente & \begin{tabular}{|lll}
$\begin{array}{l}\text { Crear producciones innovadoras y pensar } \\
\text { críticamente sobre lo aprendido }\end{array}$ & \\
\end{tabular} \\
\hline \multirow{7}{*}{ 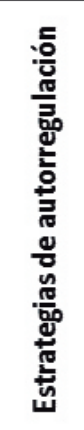 } & \multirow{4}{*}{ De la persona } & 1 & Eficacia percibida & Percepción de éxito en el estudiante \\
\hline & & 2 & Contingencia interna & \begin{tabular}{|l}
$\begin{array}{l}\text { Percepción de locus interno para el logro } \\
\text { académico }\end{array}$ \\
\end{tabular} \\
\hline & & 3 & Autonomía percibida & Autonomía en el logro de aprendizaje \\
\hline & & 4 & \begin{tabular}{|l|}
$\begin{array}{l}\text { Orientación a la } \\
\text { aprobación externa }\end{array}$ \\
\end{tabular} & $\begin{array}{l}\text { Éxito o fracaso guiado por factores externos al } \\
\text { aprendizaje }\end{array}$ \\
\hline & \multirow{2}{*}{ De la tarea } & 5 & $\begin{array}{|lll|}\begin{array}{l}\text { Orientación a la } \\
\text { tarea en sí }\end{array} & \\
\end{array}$ & $\begin{array}{l}\text { Criterios de éxito guiados por la ejecución en la } \\
\text { tarea }\end{array}$ \\
\hline & & 6 & $\begin{array}{l}\text { Orientación a las } \\
\text { metas }\end{array}$ & Orientación de éxito en el logro por metas. \\
\hline & Materiales & 7 & Utilidad & Uso eficiente en apoyo al aprendizaje \\
\hline
\end{tabular}

El segundo componente, el Inventario de Epistemología Personal, refiere a las creencias epistemológicas (concepciones individuales sobre el conocimiento). Hofer y Pintrich (2002) las conceptualizan como epistemología personal. Así, el instrumento retoma las aportaciones del modelo de Schommer-Aikins (2004), a partir de las cuales se proponen dimensiones que son representadas a lo largo de un continuo que va de creencias más ingenuas (nä̈ve) a creencias más sofisticadas (reflexivas). El Inventario de Epistemología Personal (Castañeda, Bazán y Peñalosa, 2009 y Castañeda y Peñalosa 2010a) consta de 26 ítems tipo Likert, que miden cuatro dimensiones de creencias epistemológicas (ver figura 2): estabilidad, fuente, utilidad y naturaleza del conocimiento.

Figura 2. Inventario de Epistemología Personal (IEP)

\begin{tabular}{|l|l|l|}
\hline & \multicolumn{1}{|c|}{ Creencia epistemológica } & \multicolumn{1}{c|}{ Progresión en el continuo } \\
\hline 1 & Estabilidad del Conocimiento & cierto - tentativo; estático - dinámico \\
\hline 2 & Fuente del Conocimiento & $\begin{array}{l}\text { autoridad - no autoridad; externa - } \\
\text { personal; cuestionable - no cuestionable. }\end{array}$ \\
\hline 3 & Utilidad del Conocimiento & $\begin{array}{l}\text { transferible - no transferible; visión } \\
\text { actual -visión futura }\end{array}$ \\
\hline 4 & Naturaleza del Conocimiento & $\begin{array}{l}\text { abstracta - concreta: científica - no } \\
\text { científica }\end{array}$ \\
\hline
\end{tabular}


El tercer instrumento, la Prueba de Comprensión de Textos, consta de un texto titulado Los dos reyes y los dos laberintos (Borges, 1946) y de una prueba de comprensión. El texto es de estructura narrativa con un nivel de dificultad léxico-técnica bajo, pero de dificultad sintáctica y semántica alta. La prueba de comprensión de lectura es una prueba de 20 reactivos que evalúan 10 tipos de respuesta de comprensión en dos contextos de recuperación de lo comprendido: de reconocimiento y de recuerdo. La figura 3 muestra su estructura.

Figura 3. Estructura de la prueba de comprensión de lectura

\begin{tabular}{|c|c|c|}
\hline Tarea & Contexto de & eración \\
\hline A.-idea principal & \multirow{10}{*}{ Reconocimiento } & \multirow{10}{*}{ Recuerdo } \\
\hline B.-detalle & & \\
\hline C. secuencia & & \\
\hline D.- vocabulario & & \\
\hline E.- contraste & & \\
\hline F.- deducción & & \\
\hline G.- inducción & & \\
\hline H.- resumen & & \\
\hline I.- causa- efecto & & \\
\hline J.- enumeración & & \\
\hline
\end{tabular}

\section{PROCEDIMIENTO}

Con datos de una muestra no intencional de 1361 estudiantes universitarios se construyeron bases específicas de cada constructo a ser calibrado, validado y confiabilizado. Para la calibración, se utilizó el programa IRTPro v2.1 (SSI, 2011), en sus dos opciones de análisis, la de datos dicotómicos, con modelo logístico de tres parámetros y con el modelo de respuesta graduada (Samejima, 1969) para datos politómicos. Posteriormente, se validaron los constructos subyacentes mediante el programa EQS v6.2 (Bentler, 2006), finalmente se confiabilizaron los bancos que constituyen el metaevaluador web con el mismo programa.

\section{RESULTADOS}

\section{Validación de constructo del banco de reactivos IEEA}

El banco de Estrategias de Estudio y Autorregulación (IEEA) estuvo constituido por 52 ítems iniciales que miden trece subdimensiones, seis acerca de heurísticas cognitivas y siete sobre heurísticas autorregulatorias (Castañeda, Pineda, 
Gutiérrez, Romero y Peñalosa, 2010). De ellos, se eligieron 50 ítems con valores de discriminación $>0.65$, para ser usados en el AFC de: a) el banco de Estrategias Cognitivas de Estudio, b) el de Estrategias de Autorregulación de Persona y c) el de Autorregulación de Tarea y Materiales. Los resultados son mostrados para cada subbanco, pero en lo general todos muestran validez convergente de constructo. Sus características específicas se describen a continuación.

\section{Modelo validado del banco de Estrategias Cognitivas}

El banco correspondiente a Estrategias Cognitivas, a pesar de haber obtenido un valor de $X^{2}=754.58$ y una $p=0.00$, que indica diferencias estadísticamente significativas entre el modelo saturado y el empírico, sus índices de ajuste práctico $(\mathrm{CFI}=.93$; RMSEA=.04) y su alfa de Cronbach (0.87) son adecuados, lo que muestra que no hay diferencias entre el modelo teórico y el empírico (ver figura 4). Un dato que confirma el ajuste entre modelos es el hecho de que seis de los siete índices de ajuste práctico confirman que los modelos ajustan. Por otra parte, la combinación de la varianza explicada entre factores es del $49 \%$, por lo que es razonable asumir que tanto la configuración estructural entre los factores resultantes, como la de los reactivos convergentes representan adecuadamente los constructos subyacentes al banco IEEA.

Figura 4. Modelo empírico validado del banco de Estrategias Cognitivas del IEEA en web

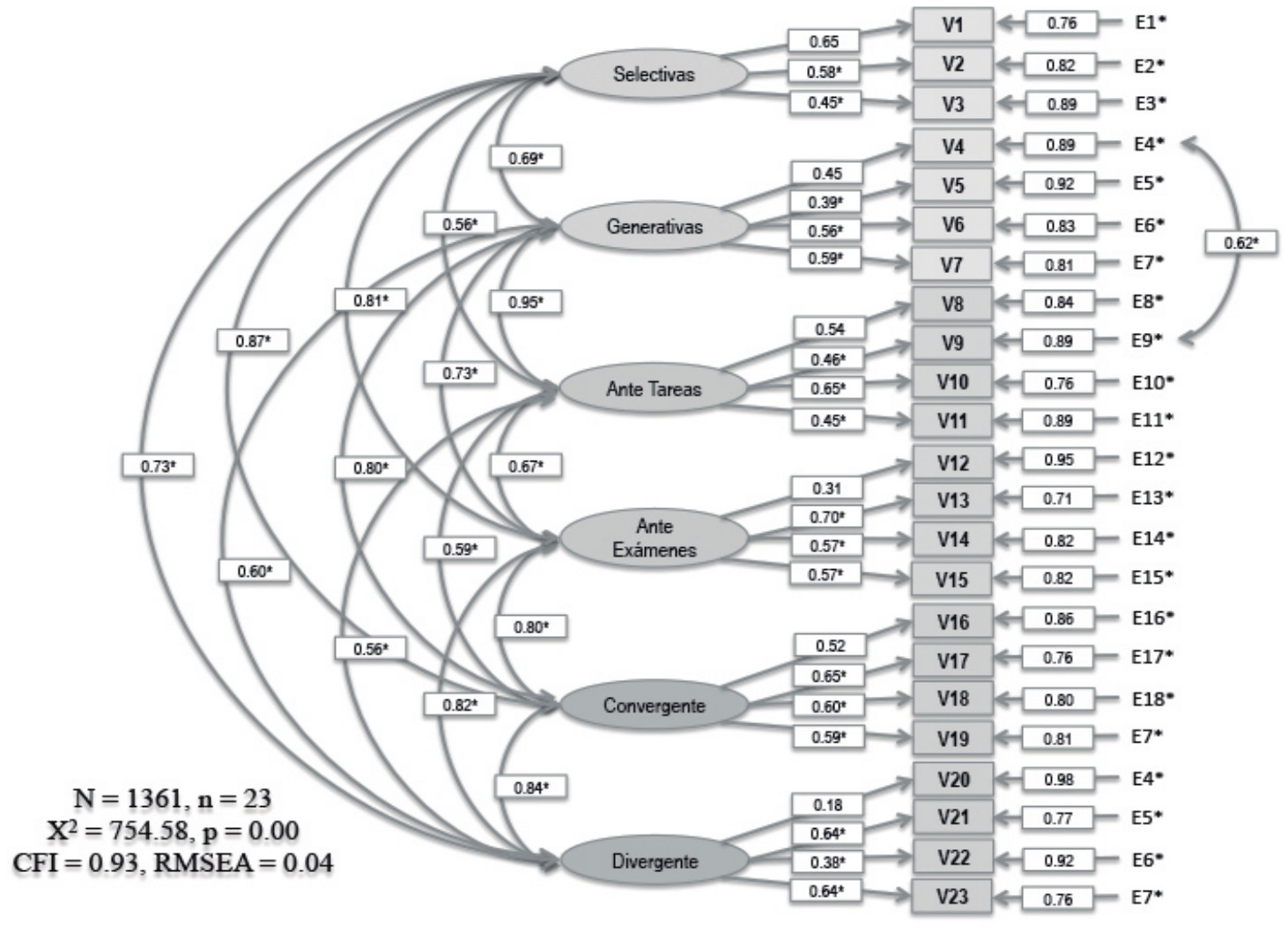


Así y considerando las características psicométricas que presenta, es posible establecer que es un banco confiable y con validez de constructo para evaluar las dimensiones hipotetizadas en el metaevaluador web. Es más, sus reactivos son capaces de discriminar a todo lo largo del continuo que se forma en cada variable latente, lo que implica el beneficio de contar con un instrumento con cualidades psicométricas estrictas, que aumentan la precisión en las mediciones y reducen la probabilidad de acumular error sistemático en las valoraciones que los estudiantes realizan sobre sus estrategias cognitivas de estudio.

Cabe hacer notar, también, la intrincada interrelación entre Estrategias de Adquisición de Información con las de Recuperación y estas con el tipo de procesamiento que se utiliza (sus correlaciones varían entre .56 y .95). Los datos reconfirman una validez convergente sólida.

\section{Modelo validado del banco de Estrategias de Autorregulación de Persona}

El segundo subbanco, el de Estrategias Autorregulatorias de Persona, también mostró un índice de ajuste práctico aceptable $(\mathrm{CFI}=.94$; RMSEA $=.05)$ y una confiabilidad adecuada de 0.82 (alfa de Cronbach). La figura 7 muestra el modelo resultante.

Figura 5. Modelo empírico validado del banco de Estrategias Autorregulatorias de Persona del IEEA en web

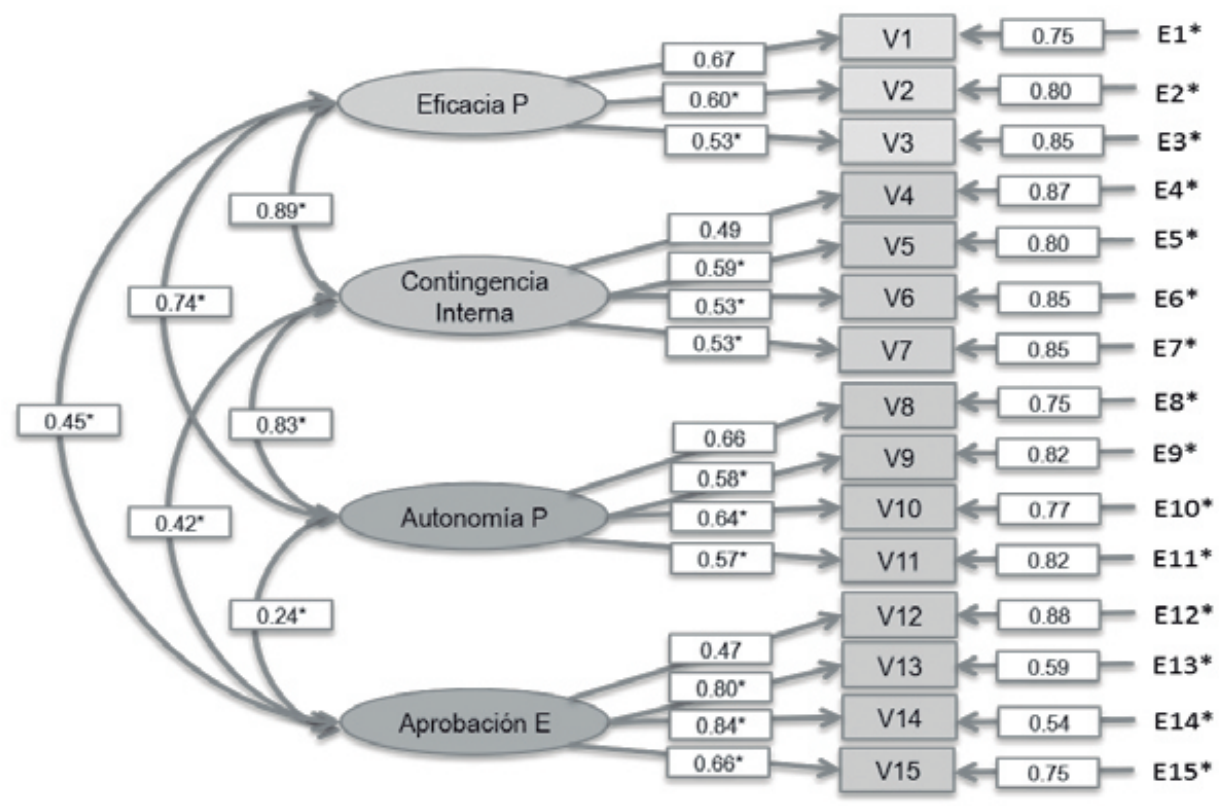

$\mathrm{N}=1361, \mathrm{n}=15$

$\mathrm{X}^{2}=413.13, \mathrm{p}=0.00$

$\mathrm{CFI}=0.94, \mathrm{RMSEA}=0.05$ 
Todas las variables observadas se asociaron a los constructos a los que hipotéticamente pertenecen (valores que van de .47 hasta .84 de cargas factoriales), lo que configura los cuatro factores que constituyen la porción de Estrategias Autorregulatorias de Persona del metaevaluador web. Cabe hacer notar también que las asociaciones entre factores muestran la intrincada interrelación entre eficacia percibida, contingencia interna y autonomía percibida como es de esperarse, pero la relación entre aprobación externa y los tres factores anteriores es apenas de .24, lo que establece la validez divergente entre él y los demás.

Las correlaciones convergentes varían entre .74 a .89 y las divergentes de .24 a .42 (todos pertenecientes a aprobación externa).

El valor de $\mathrm{X}^{2}(413.13)$ y su probabilidad $(\mathrm{p}=0.00)$ establecen que sí hay diferencias significativas. Sin embargo, es posible que este efecto también obedezca al tamaño de la muestra y a la debilidad de la $\mathrm{X}^{2}$ ante esta situación. Cabe señalar que en este modelo, también, seis de los siete índices de ajuste práctico confirman el ajuste. Por su parte, la combinación entre factores explica un $70 \%$ de la varianza.

\section{Modelo validado del banco de Estrategias de Autorregulación de Tareas y Materiales}

El tercer subbanco, el de Estrategias Autorregulatorias de Tareas y Materiales, también mostró un índice de ajuste práctico aceptable $(\mathrm{CFI}=.94$; RMSEA $=.06)$ y una confiabilidad adecuada de 0.83 (alfa de Cronbach). La figura 6 muestra el modelo resultante:

Figura 6. Modelo empírico validado del banco de Estrategias de Tarea y Materiales del IEEA en web

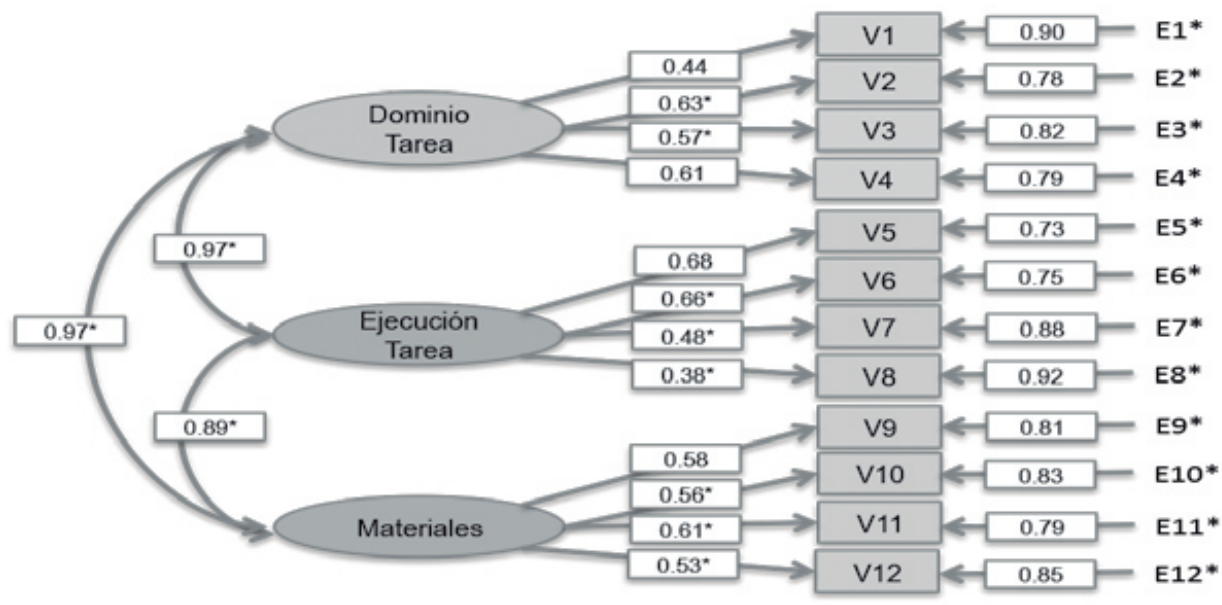

$\mathrm{N}=1361, \mathrm{n}=12$

$\mathrm{X}^{2}=298.01, \mathrm{p}=0.00$

$\mathrm{CFI}=0.94, \mathrm{RMSEA}=0.06$ 
En la Figura 8, al igual que en las dos anteriores, todas las variables observadas se asociaron a los constructos a los que hipotéticamente pertenecen, mostrando valores que van de .38 hasta .68 de cargas factoriales, lo que configura los tres factores mismos que constituyen la porción de Estrategias Autorregulatorias de Tarea y Materiales. Cabe hacer notar también que las asociaciones entre factores muestran la intrincada interrelación entre los componentes de Tarea y Materiales. Las correlaciones convergentes varían entre .89 y .97 . Es recomendable mejorar la validez divergente de esta porción.

Aun cuando el valor de $\mathrm{X}^{2}(298.01)$ y su probabilidad $(\mathrm{p}=0.00)$ establecen que sí hay diferencias significativas, como se mencionó anteriormente, esto se puede deber al tamaño de la muestra.

Cabe señalar que seis de los siete índices de ajuste práctico confirman que los modelos se ajustan. Por su parte, la combinación entre factores explica un $47 \%$ de la varianza.

\section{Validación de constructo del Inventario de Epistemología Personal (IEP)}

El segundo banco inicial mide cuatro dimensiones de epistemología personal (26 ítems distribuidos en nueve subdimensiones). Para el AFC solo se eligieron los 12 reactivos de las tres dimensiones puestas a prueba y cuyos reactivos mostraron los valores más altos de discriminación ( $>0.65)$ y más bajos de error.

El modelo a ser puesto a prueba quedó constituido entonces por tres dimensiones o constructos: a) el de Estabilidad del Conocimiento, b) el de Fuente del Conocimiento y c) el de Utilidad del Conocimiento.

La hipótesis sugiere que los reactivos que se encuentran asociados a ellas explican su estructura. Para contrastar ambos modelos, se utilizó el estadístico de $\mathrm{X}^{2}$. Se realizó el AFC utilizando el método de máxima verosimilitud (ML) y los resultados del ajuste práctico del modelo indican un CFI $=.90$ y un RMSEA de .07 . Los índices reflejan ajuste entre el modelo hipotetizado y el modelo estimado a partir de los datos empíricos. Asimismo, cabe señalar que el instrumento presentó un coeficiente Rho de confiabilidad de .76, mostrando una aceptable homogeneidad interna entre los reactivos que componen el instrumento.

La mayoría de los pesos factoriales, en las tres dimensiones validadas, alcanzaron pesos factoriales entre .36 y .73. En todos los casos los pesos factoriales fueron significativos. Dados estos resultados, es razonable afirmar que el instrumento cuenta con validez convergente. Sin embargo, las relaciones entre fuente y estabilidad del conocimiento marcan un índice de correlación alto que hace necesario mejorar la validez divergente entre estas dimensiones. Lo anterior se puede ver en la figura 7. 
Figura 7. Modelo empírico validado del banco de Epistemología Personal del IEP en web

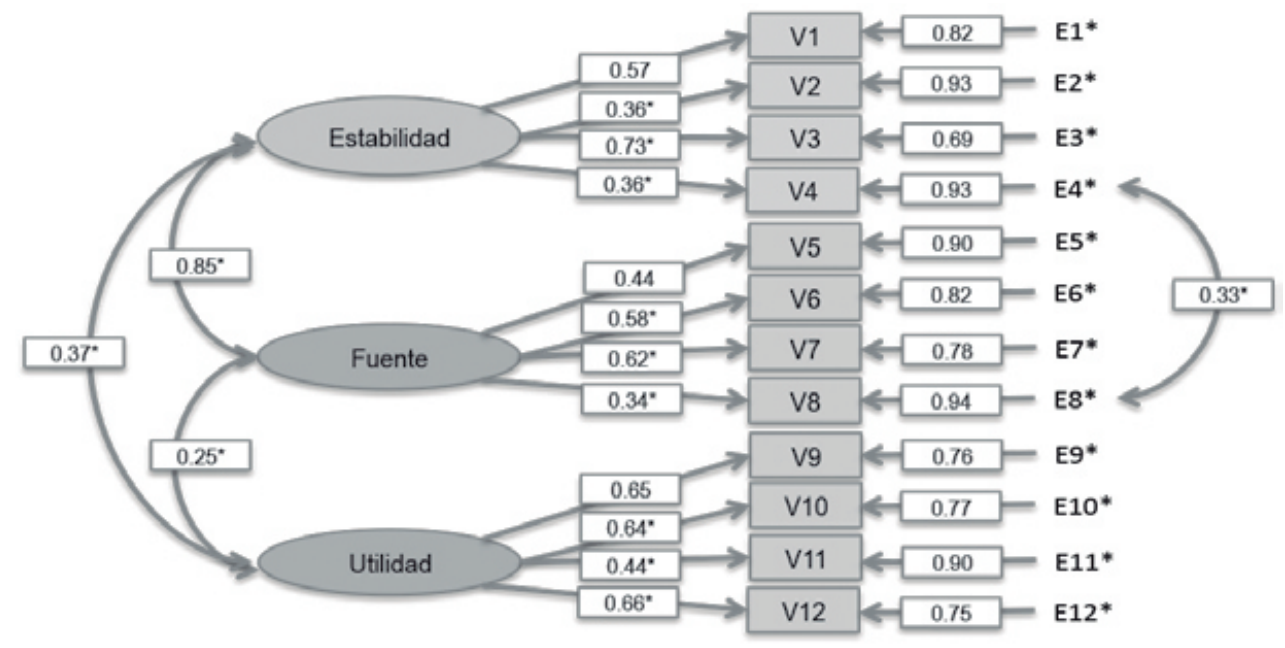

$$
\begin{gathered}
\mathrm{N}=1361, \mathrm{n}=12 \\
\mathrm{X}^{2}=336.55, \mathrm{p}=0.00 \\
\mathrm{CFI}=0.90, \text { RMSEA }=0.07
\end{gathered}
$$

Cabe señalar que seis de los siete índices de ajuste práctico confirman que los modelos se ajustan. Por su parte, la combinación entre factores explica un $53 \%$ de la varianza.

De esta forma, a partir de los análisis presentados, los bancos de reactivos son considerados con una suficiencia en su calidad psicométrica en un contexto en línea, lo que favorece su uso en la evaluación y medición de variables del estudiante referidas a las estrategias de estudio y a las creencias epistemológicas durante el aprendizaje complejo.

La utilización extensa de estos bancos de reactivos permitirá entender mejor cómo las estrategias cognitivas y autorregulatorias de los estudiantes, así como sus creencias epistemológicas, interactúan con diversos medios de entrega de la instrucción (presencial y virtual, por ejemplo) y con los más variados dominios de conocimiento (teóricos, metodológicos, técnicos, éticos, entre otros muchos).

\section{Validación de constructo del banco de reactivos derivados de la prueba de Comprensión de Textos}

Los resultados del ajuste del modelo de la prueba de comprensión de textos fueron satisfactorios e indican que el modelo teórico no difiere significativamente de la matriz de estructura empírica, según sus índices de ajuste práctico. A pesar de que la $\mathrm{X}^{2}$ indica que las diferencias entre el modelo saturado y el empírico son significativas $\left(\mathrm{X}^{2}=277.23, \mathrm{p}=0.00\right)$, el efecto depende del extenso número de sujetos utilizado en la muestra. En apoyo a la validez de la estructura factorial 
encontrada, el índice $\mathrm{CFI}=.96$ con un $\mathrm{RMSEA}=0.03$, muestra que el ajuste entre ambos modelos es óptimo. Además se presentó un alfa de Cronbach de 0.78, mostrando una aceptable homogeneidad interna entre los reactivos. (ver figura 8).

Figura 8. Modelo empírico validado para la prueba de Comprensión de Textos Web

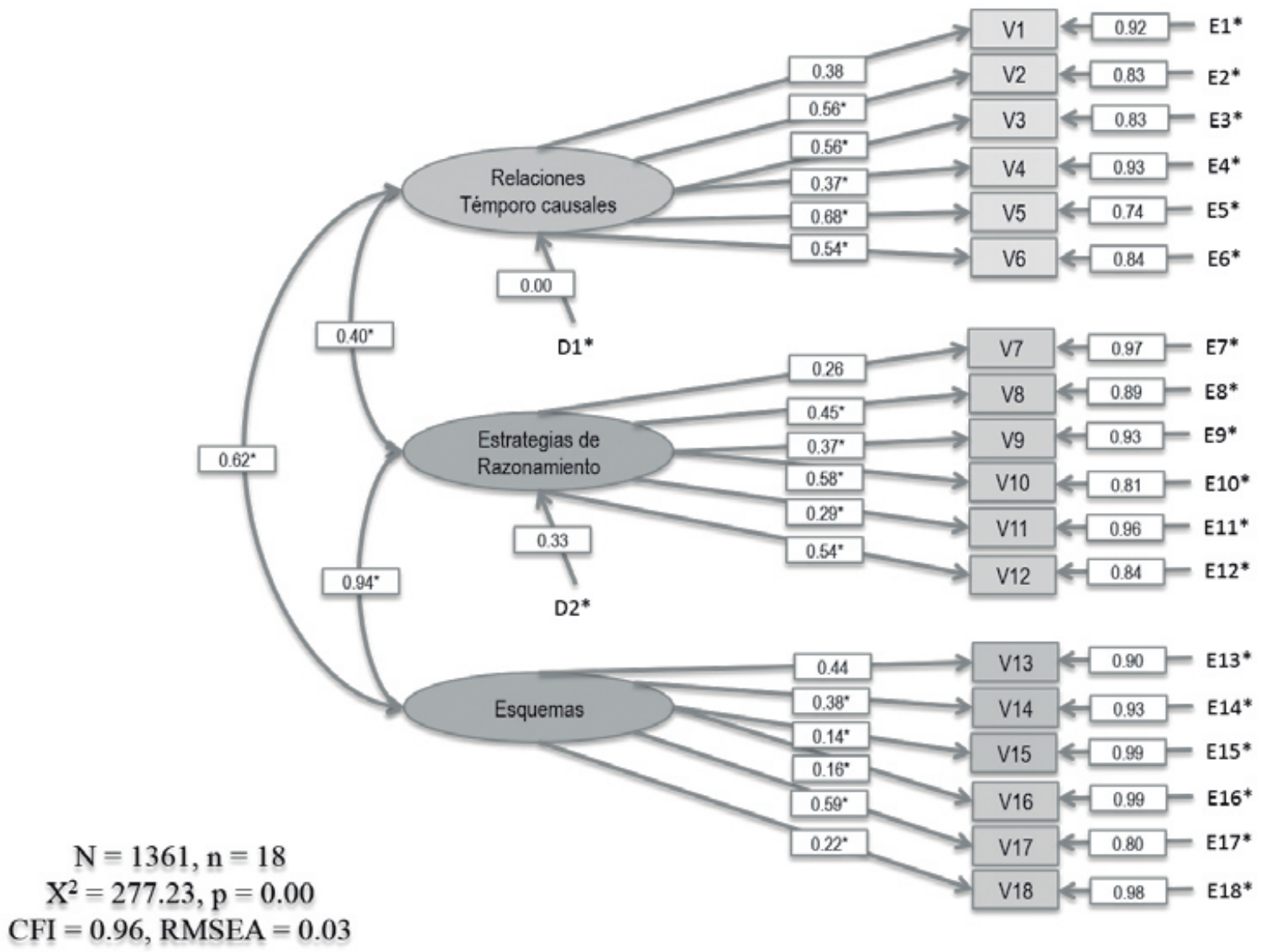

Así, es plausible pensar que este banco de reactivos es capaz de representar adecuadamente las tres dimensiones hipotetizadas para medir la comprensión de textos en esta prueba: a) entender las relaciones témporo-causales contenidas en el texto, b) aplicar estrategias de razonamiento inductivo, deductivo y analógico para entender la información vertida en el texto y c) comprender esquemas de conocimiento de simples a complejos en el contenido del texto. La validez convergente en toda la prueba es buena. Habrá que mejorar la validez divergente entre esquemas y relaciones témporo-causales (.62) y entre esquemas y estrategias de razonamiento (.94).

En las tres dimensiones validadas, aun cuando se alcanzó una validez convergente, los pesos factoriales oscilan entre .14 y .68. Es importante mencionar que los reactivos con pesos factoriales menores de 0.3 y errores cercanos a 1.00 serán modificados para mejorar sus pesos factoriales. 


\section{DISCUSIÓN}

Los resultados muestran validez convergente y ajuste práctico entre todos los modelos teóricos y los empíricos puestos a prueba en cada uno de los análisis factoriales confirmatorios. Se confirmó la validez de los constructos subyacentes a los tres bancos que constituyen el metaevaluador web.

De esta forma, podemos establecer que al utilizar herramientas de análisis multivariado en la construcción de esta innovación tecnológica, se controló y redujo el error, se aumentó la discriminación de los reactivos entre niveles de dificultad, se validaron los constructos y se estimó la homogeneidad entre ítems.

\section{CONCLUSIONES GENERALES}

Este trabajo presenta dos aportaciones importantes. La primera se refiere a los procesos de construcción, calibración y validación de los bancos de reactivos capaces de aportar datos sin sesgo, donde el error es controlado estadísticamente y los constructos subyacentes son validados mediante procedimientos estadísticos multivariados y multidimensionales. Particularmente, ante fenómenos como el de agencia académica que, por su naturaleza compleja y situacional, son elusivos a una medición orientada al rasgo.

La segunda aportación tiene que ver con su implementación en una plataforma web que permite la optimización de recursos durante los procesos de aplicación, calificación y reporteo, pero, sobre todo, las facilidades que le brinda al investigador y al tomador de decisiones educativas al generar evidencias empíricas que les permitan entender cómo interactúan estos componentes en diferentes contenidos y contextos. De esta manera, se pueda incidir en su diagnóstico y fomento a partir de una base objetiva de detección estandarizada y psicometrada.

\section{NOTA DE RECONOCIMIENTO}

Reconocemos el apoyo por parte de la Universidad Autónoma Metropolitana, al Consejo Nacional de Ciencia y Tecnología (Conacyt) y las instituciones participantes, quienes facilitaron el desarrollo de la investigación.

\section{REFERENCIAS BIBLIOGRÁFICAS}

Baxter, M. (1992). Knowing and reasoning in college: Gender-related patterns in Students intellectual development. San Francisco: Jossey Bass.

Belenky, M., Clinchy, B., Goldberger, N. \& Tarule, J. (1986). Women's Ways of Knowing: The Development of Self, Voice, and Mind. New York: Basic Books.

Bentler, P. (2006). EQS 6.1 for Windows (Build 90) [Software de Computadora]. Encino, CA: Multivariate Software, Inc. 
Castañeda, S. (2007). Resignificando el aprendizaje en la Educación Superior. En Z. Monroy y P. Fernández (Eds.), Lenguaje, Significado y Psicología (pp. 35-46). México: UNAM.

Castañeda, S., Bazán, A. y Peñalosa, E. (2009). Validación y significancia de constructo de la evaluación de egreso de psicólogos educativos. Nuevos Paradigmas. Revista Psicológica de Actualización Profesional, 2(1), 24-46. Perú.

Castañeda-Figueiras, S. y Peñalosa, E. (2010). Validando constructos en epistemología personal. Revista Mexicana de Psicología, Volumen 27, 1, 65-75.

Castañeda, S. (2012). El Sentido de Agencia en el Aprendizaje de Contenidos Teóricos. Resúmenes del XX Congreso Mexicano de Psicología. México. pp. 563-564.

Castañeda, S, Peñalosa, E. y Austria, F. (2014). Perfiles agentivos y no agentivos en la formación del psicólogo. México: UNAM-CONACyT. ISBN-e 978607-02-5996-8.

Castañeda, S., Pérez, I. y Peñalosa, E. (2014). Evaluando componentes de Agencia Académica en la web. PSICUMEX, 4(1), 98-117.

Castañeda, S., Pineda, M., Gutiérrez, E., Romero N. y Peñalosa, E. (2010). Construcción de instrumentos de estrategias de estudio, autorregulación y epistemología personal. Validación de constructo. Revista Mexicana de Psicología, 27(1), 77-85.

Hofer, B. \& Pintrich, P. (2002). Personal epistemology: The psychology of beliefs about knowledge and knowing. Mahwah, NJ: Lawrence Erlbaum.

Kuhn, D. (1991). The skills of argument. Cambridge: Cambridge University Press.

Kuhn, D., Cheney, R. \& Weinstock, M. (2000). The development of epistemological understanding. Cognitive Development, 15, 309-328.

Perry, W. (1970). Forms of Intellectual and Ethical Development in the College Years: A Scheme. New York: Holt, Rinehart and Winston.

Samejima, F. (1969). Estimation of Latent Ability Using a Response Pattern of Graded Scores (Psychometric Monograph No. 17). Richmond, VA: Psychometric Society.

SSI (2011). Scientific Software International (2011). IRTPRO 2.1 Flexible Professional Item Response Theory Modeling for Patient- Reported Outcomes [Software de Computadora]. Sc.

Schommer, M. (1990). Effects of beliefs about the nature of knowledge on comprehension. Journal of Educational Psychology, 82, 498-504.

Schommer-Aikins, M. (2004) Explaining the epistemological belief system: Introducing the embedded systemic model and coordinated research approach. Educational Psychologist, 39, 19-29. 
Scientific Software International (2011). IRTPRO 2.1 Flexible Professional Item Response Theory Modeling for Patient- Reported Outcomes [Software de Computadora]. Sc. 